\title{
Dietary Oxidative Balance Scores and Biomarkers of Inflammation among Individuals with and without Chronic Kidney Disease
}

\author{
Kristin J. Marks ${ }^{a}$ Terryl J. Hartman ${ }^{a}$ Suzanne E. Judd ${ }^{b}$ Titilayo O. Iloric \\ Katharine L. Cheung ${ }^{d}$ David G. Warnock ${ }^{b}$ Orlando M. Gutiérrez ${ }^{b}$ \\ Michael Goodman ${ }^{a}$ Mary Cushman ${ }^{d}$ William M. McClellan ${ }^{a}$

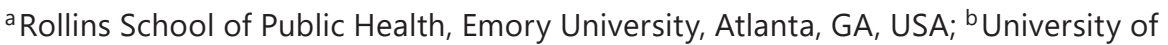 \\ Alabama at Birmingham, Birmingham, AL, USA; ' University of Arizona, Tucson, AZ, USA; \\ dUniversity of Vermont, Burlington, VT, USA
}

\section{Keywords}

Oxidative balance $\cdot$ Pro-oxidant $\cdot$ Antioxidant $\cdot$ Chronic kidney disease $\cdot$ Inflammatory markers

\begin{abstract}
Background: Oxidative stress and inflammation are proposed mechanisms of nonspecific kidney injury and progressive kidney failure. Higher dietary oxidative balance scores (OBS) are associated with lower prevalence of chronic kidney disease (CKD). Methods: We investigated the association between OBS and biomarkers of inflammation using data from the Reasons for Geographic and Racial Differences in Stroke (REGARDS) study. Nutrient estimates from the Block Food Frequency Questionnaires were used to define tertiles of 11 pro- and antioxidant factors. Points for each OBS component were summed, with a higher score indicating predominance of antioxidant exposures. Multivariable linear regression models were used to estimate the association between OBS and biomarkers of inflammation (interleukin-6 [IL-6], interleukin-8 [IL-8], interleukin-10 [IL-10], fibrinogen, C-reactive protein [CRP], white blood cell count, and cystatin C). An interaction term was included to determine if associations between OBS and inflammatory markers differed between individuals with and without CKD. Results: Of 682 participants, $22.4 \%$ had CKD. In adjusted models, OBS was associated with CRP and IL-6. For every 5-unit increase in OBS, the CRP concentration was $-15.3 \%$ lower (95\% Cl: -25.6 ,
\end{abstract}


-3.6). The association of OBS with IL-6 differed by CKD status; for every 5 -unit increase in OBS, IL- 6 was $-10.7 \%$ lower $(95 \% \mathrm{Cl}:-16.3,-4.7)$ among those without CKD, but there was no association among those with $\operatorname{CKD}(p=0.03)$. Conclusion: This study suggests that a higher OBS is associated with more favorable levels of IL- 6 and CRP, and that the association of OBS and IL- 6 may be modified by CKD status.

(C) 2018 The Author(s)

Published by S. Karger AG, Basel

\section{Introduction}

Oxidative stress, defined as an imbalance of pro-oxidant and antioxidant exposures wherein the effects of the pro-oxidants exceed the effects of the antioxidants, is a potential source of cellular damage [1]. Oxidative stress is closely related to, and is both a cause and consequence of, inflammation. Oxidative stress has pro-inflammatory effects through activation of nuclear factor-kappa $B$, which increases the expression of chemokines, cytokines, and cell adhesion molecules [2-5].

Oxidative stress and inflammation are proposed mechanisms of nonspecific kidney injury and progressive kidney failure [6,7]. Studies have found that chronic kidney disease (CKD) is associated with increased levels of reactive oxygen and nitrogen species [6-9]; this occurs in early CKD and may be a factor in a progressive decline in the glomerular filtration rate and vascular complications $[8,10,11]$. Further, individuals with CKD and compromised nutritional status show more signs of oxidative stress than well-nourished individuals $[12,13]$.

There is evidence that diet, medication, and lifestyle are important exogenous sources contributing to the body's oxidative balance [6]. However, it has been difficult to show associations between individual dietary antioxidants and kidney injury, as the effects may be modest, correlated, and may involve biological interactions across multiple factors [14]. Evidence is emerging that a dietary score based on multiple oxidative stress-influencing nutrients may capture the complex interactions between individual pro- and antioxidants, and is associated with disease states [6,15-18], such as CKD [19].

It was previously reported that a higher oxidative balance score (OBS), defined by a composite estimate of the overall pro- and antioxidant exposure status in an individual, is associated with lower prevalence of CKD among participants of the Reasons for Geographic and Racial Differences in Stroke (REGARDS) study [19]. After multivariable adjustment, prevalent CKD remained associated with decreased OBS; and while the association with end-stage renal disease was not statistically significant, a reduced risk of end-stage renal disease was suggested with higher levels of OBS. It was also previously reported that REGARDS participants with higher OBS were more likely to have favorable levels of surrogate markers for inflammation, such as decreased C-reactive protein (CRP) and lower total white blood cell (WBC) count [20]. Similar observations have been reported elsewhere [4].

This analysis extends upon previous findings [4, 19, 20] by examining additional biomarkers of inflammation beyond CRP and WBC that might provide clues as to different pathways of inflammation, and does so in the context of CKD. The purpose of this study is to examine the association between OBS and a wider range of inflammatory biomarkers, and whether this association differs in persons with and without CKD. 
Fig. 1. Flowchart of participants in the REGARDS study who were included in the analysis. eGFR, estimated glomerular filtration rate; ACR, albumin/creatinine ratio.

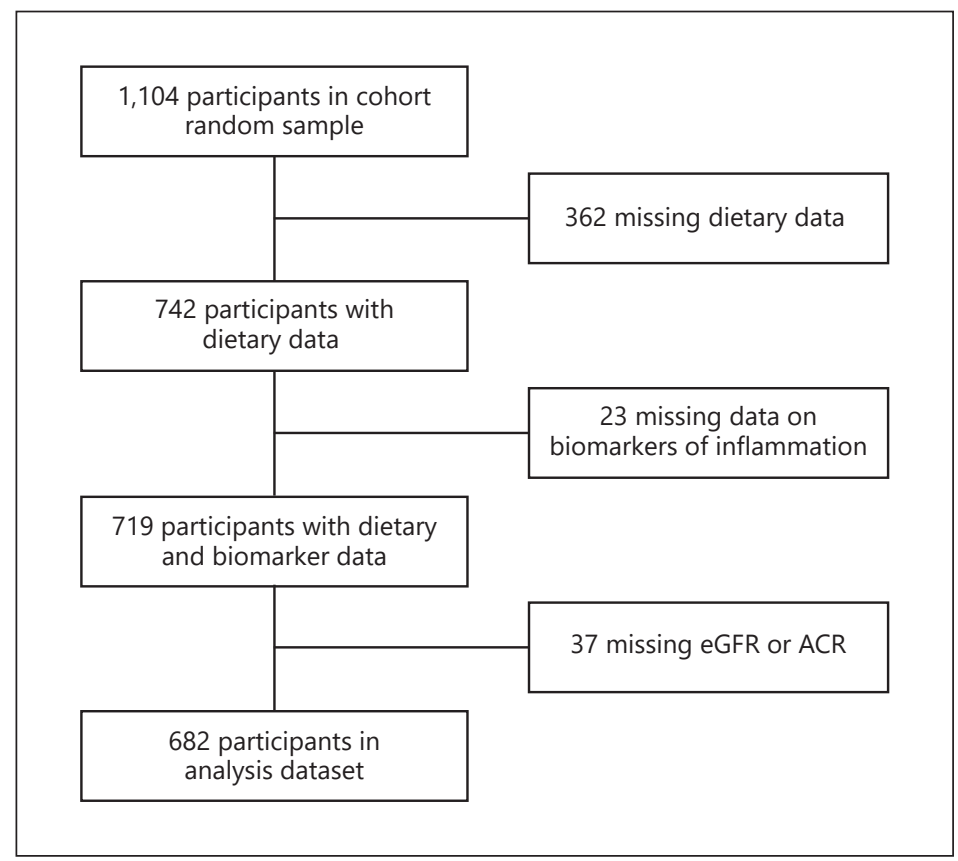

\section{Methods}

\section{Study Design}

The REGARDS study is a national, population-based prospective cohort study that aims to examine reasons for variation in stroke incidence in the United States. Recruitment and data collection are described in detail elsewhere [21]. Briefly, between January 2003 and October 2007, 30,239 volunteers aged 45 years and older were randomly selected and recruited, with planned equal recruitment of men and women. There was oversampling of African Americans and residents of the 8 southeastern states with a higher stroke mortality than in the rest of the US. The 8 states are divided into the stroke "buckle" (coastal plain regions of Georgia, North Carolina, and South Carolina) and the "belt" (the remaining area of Georgia, North Carolina, and South Carolina, plus Mississippi, Alabama, Tennessee, Louisiana, and Arkansas) [22].

Trained personnel used a computer-assisted telephone survey to collect data on demographic factors (e.g., age, race, sex), socioeconomic factors (e.g., household income, education, marital status), and medical history. Next, a health professional visited the participants' homes to collect anthropomorphic variables as well as blood and urine samples. The REGARDS study was approved by the institutional review boards of the participating institutions, and all participants provided written informed consent before completion of the in-home study visit.

The 1998 Block Food Frequency Questionnaire (Block 98 FFQ; Nutrition Quest, Berkeley, CA, USA) was left with the participants to be self-administered and returned. The FFQ is comprised of 107 items that assess dietary intake over the previous year. The Block $98 \mathrm{FFQ}$ has been extensively validated using multiple food records in diverse populations in the United States, and reliably estimates the intake of nutrients [23-26]. The FFQ has been described in detail elsewhere [22,27]. Completed FFQs were scanned and sent to Nutrition Quest $^{\mathrm{TM}}$ (Berkeley, CA, USA) for the analysis of the nutrition content. The dietary data collected using the FFQ were used to calculate the OBS. 
Table 1. Dietary oxidative balance score (OBS) components ${ }^{\mathrm{a}}$

\begin{tabular}{|c|c|}
\hline 1. Cryptoxanthin & $0=\operatorname{low}(1$ st tertile), $1=$ medium $(2$ nd tertile $), 2=$ high $(3 \mathrm{rd}$ tertile $)$ \\
\hline \multicolumn{2}{|r|}{ 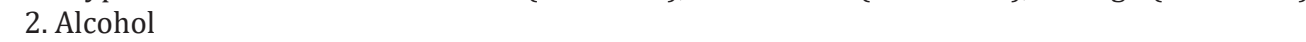 } \\
\hline Female & $0=8+$ drinks $/$ week, 1 = 1-7 drinks/week, $2=<1 \mathrm{drink} /$ week \\
\hline Male & $0=15+$ drinks $/$ week, 1 = 1-14 drinks $/$ week, $2=<1$ drink $/$ week \\
\hline 3. PUFA & $0=$ high $(3$ rd tertile), $1=$ medium ( 2 nd tertile), $2=$ low ( 1 st tertile) \\
\hline 4. Total iron ${ }^{\mathrm{b}}$ & $0=$ high $(3 r d$ tertile), $1=$ medium ( 2 nd tertile), $2=$ low ( 1 st tertile) \\
\hline 5. Total vitamin C & $0=\operatorname{low}(1$ st tertile), $1=$ medium $(2$ nd tertile $), 2=$ high $(3 r d$ tertile $)$ \\
\hline 6. Total $\alpha$-carotene & $0=\operatorname{low}(1$ st tertile), $1=$ medium $(2$ nd tertile $), 2=$ high $(3 \mathrm{rd}$ tertile $)$ \\
\hline 7. Total $\beta$-carotene & $0=\operatorname{low}(1$ st tertile), $1=$ medium $(2$ nd tertile $), 2=$ high $(3 r d$ tertile $)$ \\
\hline 8. Total vitamin $\mathrm{E}$ & $0=$ low ( 1 st tertile), $1=$ medium ( 2 nd tertile), $2=$ high ( $3 r d$ tertile) \\
\hline 9. Total selenium & $0=\operatorname{low}(1$ st tertile), $1=$ medium $(2$ nd tertile $), 2=$ high $(3 \mathrm{rd}$ tertile $)$ \\
\hline 10. Lutein & $0=\operatorname{low}(1$ st tertile), $1=$ medium ( 2 nd tertile), $2=$ high $(3 \mathrm{rd}$ tertile) \\
\hline 11. Lycopene & $0=$ low (1st tertile), $1=$ medium ( 2 nd tertile), $2=$ high $(3 r d$ tertile) \\
\hline
\end{tabular}

PUFA, polyunsaturated fatty acid. ${ }^{a}$ Low, medium, and high categories correspond to sex-specific 1st, 2nd, and 3rd tertiles, adjusted for total energy intake via the residual method. ${ }^{\mathrm{b}}$ Total, dietary and supplemental.

The data for the present analysis come from a case-cohort study designed to investigate biomarkers of stroke and cognitive impairment within the REGARDS study [28, 29]. The cohort random sample included 1,104 participants selected using stratified random sampling to ensure sufficient representation of high-risk groups ( $50 \%$ blacks, $50 \%$ whites; $50 \%$ women, $50 \%$ men) and age groups (20\% aged $45-54$ years, $20 \%$ aged $55-64$ years, $25 \%$ aged $65-74$ years, $25 \%$ aged $75-84$ years, and $10 \%$ aged $\geq 85$ years).

\section{Population}

From the cohort random sample $(n=1,104)$, we excluded 362 participants who did not have data from the Block FFQ or were missing data on OBS components (Fig. 1). We also excluded 23 participants who did not have data on any of the 7 biomarkers of inflammation, and 37 participants with missing albumin/creatinine ratio (ACR) or missing estimated glomerular filtration rate (eGFR). A total of 682 participants were included in the final analysis. Excluded individuals were more likely to be black, diabetic, have a lower income, and have a lower level of education.

\section{Oxidative Balance Scores}

The exposure of interest, OBS, incorporates information on 11 a priori defined pro- and antioxidant factors (Table 1) [15, 30]. The pro-oxidants included were polyunsaturated fatty acid, total (food and supplement) iron, and use of alcohol. The antioxidants considered were total selenium, total vitamin $\mathrm{C}$, total vitamin $\mathrm{E}$, total $\alpha$-carotene, total $\beta$-carotene, lutein, lycopene, and cryptoxanthin [15]. Smoking, which was included in the original OBS, was excluded here as it is a strong risk factor for CKD [31-36]; furthermore, the use of nonsteroidal anti-inflammatory drugs (NSAIDs) and aspirin was excluded as well because avoidance of NSAIDs is recommended for most patients with CKD [37-40].

Continuous dietary variables were adjusted for total energy intake via the residual method of Willett and Stampfer [41]. The continuous dietary variables representing prooxidants (polyunsaturated fatty acid and iron) and antioxidants (selenium, vitamin C, vitamin $\mathrm{E}, \alpha$-carotene, $\beta$-carotene, lutein, lycopene, and cryptoxanthin) were categorized as low, medium, and high based on each exposure's sex-specific tertiles. For antioxidants, the first through third tertiles were assigned 0-2 points, while pro-oxidants were scored in the reverse ( 0 points for the third tertile and 2 points for the first tertile). As for alcohol consumption, 
nondrinkers received 2 points, moderate drinkers (1-7 drinks/week for women and 1-14 drinks/week for men) received 1 point, and heavy drinkers ( $>7$ drinks/week for women and $>14$ drinks/week for men) received 0 points (Table 1). The points for each component were summed to calculate the overall OBS where lower (less favorable) and higher (more favorable) total scores indicate predominance of pro- and antioxidant exposures, respectively.

\section{Biomarkers of Inflammation}

Biomarkers of inflammation under study were interleukin-6 (IL-6), interleukin-8 (IL-8), interleukin-10 (IL-10), fibrinogen, CRP, WBC count, and cystatin C (Cys C). Blood was collected during the in-home examination after an 8- to 10-h fast; sample processing and validation of the laboratory data have been previously reported [42]. CRP and Cys C were measured by particle-enhanced immunonephelometry using the BNII nephelometer (N High Sensitivity CRP, N Latex Cystatin C; Dade Behring) [43] and WBC count was measured using an automated analyzer (Beckman Coulter, Inc., Fullerton, CA, USA) [44] at the University of Vermont Laboratory for Clinical Biochemistry Research.

IL-6, IL-8, IL-10, and fibrinogen were measured in the cohort random sample in August 2012 at the University of Vermont Laboratory for Clinical Biochemistry Research. IL-6 was measured by ultra-sensitive ELISA (Quantikine HS Human IL-6 Immunoassay; R and D Systems, Minneapolis, MN, USA), IL-8 was measured by the Human Serum Adipokine Panel B LINCOplex Kit (Linco Research, Inc.; St. Charles, MO, USA), IL-10 was measured using the Milliplex MAP Human Cardiovascular Disease (CVD) Panel 3 (Millipore Corporation; Billerica, MA, USA) run as a single-plex assay, and fibrinogen was measured using the BNII nephelometer (N Antiserum to Human Fibrinogen; Siemens Healthcare Diagnostics, Deerfield, IL, USA).

\section{Chronic Kidney Disease}

CKD was defined as the joint distribution of ACR and eGFR as described in current clinical practice guidelines [45] (eGFR $<60 \mathrm{~mL} / \mathrm{min} / 1.73 \mathrm{~m}^{2}$ according to the Chronic Kidney Disease Epidemiology Collaboration equation [46] or albuminuria $\geq 30 \mathrm{mg} / \mathrm{g}$ ).

\section{Covariates}

Age, sex, race, smoking, physical activity, income, educational attainment, aspirin use, statin use, NSAID use, and diabetes were self-reported. Region was classified as stroke belt, stroke buckle, or nonbelt. Energy and protein intakes were obtained from the FFQ and analyzed as continuous variables. Weight (kilograms), height (meters), and waist circumference (centimeters) were measured during the in-home visit following a standardized protocol. BMI was calculated as weight $(\mathrm{kg}) /$ height $\left(\mathrm{m}^{2}\right)$. Waist circumference was measured with a tape measure at a point midway between the lowest rib and the iliac crest with the subject standing.

\section{Statistical Analyses}

All data analyses were performed using SAS 9.4 (Cary, NC, USA). Characteristics of the study population were reported overall and across OBS intervals. Differences in characteristics across the OBS intervals were tested using analysis of variance for normally distributed continuous variables, Kruskal-Wallis test for skewed variables, and $\chi^{2}$ statistics for categorical variables.

OBS was the independent variable of interest and was treated as a continuous variable or in intervals (interval 1 was set to range in score from 0 to 5 , interval 2 from 6 to 9 , interval 3 from 10 to 13 , interval 4 from 14 to 17 , and interval 5 from 18 to 22). The dependent variables of interest were IL-6, IL-8, IL-10, fibrinogen, CRP, WBC count, and Cys C; dependent 
Marks et al.: Oxidative Balance Score, Inflammation and CKD

Table 2. Characteristics of participants in the REGARDS cohort random sample by oxidative balance score (OBS) interval (higher intervals represent higher antioxidant exposure) $(n=682)$

\begin{tabular}{|c|c|c|c|c|c|c|}
\hline \multirow[t]{2}{*}{ Characteristic $^{\mathrm{a}}$} & \multirow[t]{2}{*}{ All } & \multicolumn{5}{|l|}{ OBS } \\
\hline & & $\begin{array}{l}\text { interval } 1 \\
0-5\end{array}$ & $\begin{array}{l}\text { interval } 2 \\
6-9\end{array}$ & $\begin{array}{l}\text { interval } 3 \\
10-13\end{array}$ & $\begin{array}{l}\text { interval } 4 \\
14-17\end{array}$ & $\begin{array}{l}\text { interval } 5 \\
18-22\end{array}$ \\
\hline$n$ & 682 & 22 & 161 & 272 & 195 & 32 \\
\hline Age, years & $66.9(11.9)$ & $61.3(10.6)$ & $65.9(12.6)$ & $67.0(12.1)$ & $68.7(11.0)$ & $64.0(11.3)^{\mathrm{b}}$ \\
\hline Female, \% & 51.3 & 54.6 & 49.7 & 51.1 & 50.8 & 62.5 \\
\hline White, $\%$ & 56.3 & 40.9 & 59.0 & 55.5 & 54.4 & 71.9 \\
\hline Current smoking, \% & 13.9 & 31.8 & 20.5 & 13.2 & 6.1 & $21.9^{\mathrm{b}}$ \\
\hline \multicolumn{7}{|l|}{ Physically inactive } \\
\hline$\leq 3$ times/week, $\%$ & 71.7 & 81.8 & 78.0 & 70.3 & 66.8 & 75.0 \\
\hline Income < USD 20k, \% & 15.3 & 13.6 & 13.7 & 16.9 & 13.9 & 18.8 \\
\hline Education $<$ high school, \% & 12.5 & 13.6 & 11.8 & 14.3 & 10.8 & 9.4 \\
\hline Hypertension, $\%$ & 56.9 & 77.3 & 50.3 & 59.4 & 57.3 & 53.1 \\
\hline Diabetes, $\%$ & 19.7 & 18.2 & 16.9 & 21.0 & 22.2 & 9.4 \\
\hline Waist circumference, $\mathrm{cm}$ & $94.7(15.0)$ & $96.9(12.3)$ & $94.6(15.3)$ & $95.9(15.3)$ & $94.0(15.1)$ & $88.7(9.7)$ \\
\hline BMI & $28.7(5.9)$ & $30.3(3.8)$ & $28.1(5.9)$ & $29.3(6.3)$ & $28.5(5.5)$ & $27.6(5.1)$ \\
\hline Calories, kcal & $1,766(761)$ & $2,052(826)$ & $1,929(795)$ & $1,719(797)$ & $1,634(631)$ & $1,960(760)^{\mathrm{b}}$ \\
\hline $\mathrm{eGFR}, \mathrm{mL} / \mathrm{min} / 1.73 \mathrm{~m}^{2}$ & $87(70-99)$ & $90(67-110)$ & $88(69-100)$ & $86(69-98)$ & $86(72-97)$ & $92(72-104)$ \\
\hline $\mathrm{ACR}, \mathrm{mg} / \mathrm{g}$ & $7.3(4.5-14.6)$ & $5.0(3.3-11.5)$ & $7.0(4.1-12.7)$ & $7.2(4.9-14.0)$ & $7.7(4.4-18.4)$ & $9.3(4.4-23.7)$ \\
\hline $\mathrm{CKD}^{\mathrm{c}, \%}$ & 22.4 & 18.2 & 19.3 & 23.9 & 24.6 & 15.6 \\
\hline IL-6, pg/mL & $2.8(2.0-4.3)$ & $3.2(2.3-4.4)$ & $2.9(2.1-4.6)$ & $2.9(2.0-4.3)$ & $2.8(1.9-4.1)$ & $2.5(1.8-3.9)$ \\
\hline IL-8, pg/mL & $2.5(1.8-3.5)$ & $2.4(2.1-3.0)$ & $2.4(1.7-3.8)$ & $2.5(1.7-3.7)$ & $2.6(1.9-3.3)$ & $2.2(1.7-3.3)$ \\
\hline IL-10, pg/mL & $9.3(6.4-13.4)$ & $9.1(5.4-14.5)$ & $9.3(6.6-13.3)$ & $9.4(6.4-13.8)$ & $9.4(6.5-12.8)$ & $7.8(6.4-12.6)$ \\
\hline Fibrinogen, mg/dL & $388(337-455)$ & $370(333-451)$ & $393(340-473)$ & $391(339-447)$ & $382(335-443)$ & $383(325-482)$ \\
\hline $\mathrm{CRP}, \mathrm{mg} / \mathrm{L}$ & $2.0(0.9-4.9)$ & $3.2(1.7-11.8)$ & $2.2(0.9-5.1)$ & $2.1(0.9-5.2)$ & $1.8(0.8-4.4)$ & $1.8(0.7-5.7)$ \\
\hline WBC count, $1,000 / \mu \mathrm{L}$ & $5.5(4.6-6.7)$ & $6.2(5.1-7.9)$ & $5.6(4.8-6.7)$ & $5.6(4.5-6.6)$ & $5.5(4.6-6.8)$ & $5.6(4.8-6.1)$ \\
\hline Cys C, mg/L & $1.0(0.8-1.1)$ & $0.9(0.8-1.1)$ & $1.0(0.8-1.1)$ & $1.0(0.8-1.2)$ & $1.0(0.8-1.2)$ & $0.9(0.8-1.1)$ \\
\hline
\end{tabular}

OBS, oxidative balance score; BMI, body mass index; eGFR, estimated glomerular filtration rate; ACR, albumin/creatinine ratio; CKD, chronic kidney disease; IL, interleukin; CRP, C-reactive protein; WBC, white blood cell; Cys C, cystatin C. ${ }^{\text {a }}$ Values for age, waist circumference, BMI, and calories are reported as mean ( \pm SD). Values for eGFR, ACR, IL-6, IL-8, IL-10, fibrinogen, CRP, WBC count, and Cys C are reported as median (IQR). Sex, race, current smoking status, income, education, hypertension, diabetes, and chronic kidney disease (CKD) are reported as percent. ${ }^{\mathrm{b}} p<0.05$ based on the ANOVA, Kruskal-Wallis, or $\chi^{2}$ tests, as appropriate. ${ }^{\mathrm{c}} \mathrm{CKD}$ was defined as eGFR $<60 \mathrm{~mL} / \mathrm{min} / 1.73 \mathrm{~m}^{2}$ or albuminuria $\geq 30 \mathrm{mg} / \mathrm{g}$.

variables that were not normally distributed were log-transformed to meet the assumptions of normality. The first OBS interval, representing a predominance of pro-oxidants, was used as the reference group when the OBS distribution was divided into intervals.

A set of potential confounding variables was identified a priori, based on previously published literature and biological plausibility, for consideration in multivariable linear regression models used to estimate the association between OBS and biomarkers of inflammation. The final model was selected after removing covariates from the full model that did not satisfy our a priori change-in-estimate criterion of $10 \%$. The final model, with weighting to account for the sampling design of the cohort random sample, was adjusted for age, sex, race, region, smoking status, BMI, protein intake, energy intake, NSAID, aspirin, and statin use. An interaction term between OBS and CKD (yes/no) was included in models to determine if the associations between OBS and biomarkers of inflammation were comparable for individuals with and without CKD. The trend tests for OBS (from lower to higher intervals) were conducted in final multivariable models by assigning the median value from each interval of OBS and modeling this as a continuous variable.

\section{Results}

There were 682 participants included in the analysis. On average, participants were 66.9 (standard deviation: 11.9 ) years old, $51.3 \%$ were women, and $56.3 \%$ were white. Participants, on average, were overweight (BMI: 28.7), and 57\% were hypertensive while a little less 
Marks et al.: Oxidative Balance Score, Inflammation and CKD

Table 3. Adjusted models ${ }^{\mathrm{a}}$ showing the association between OBS (continuous and interval) and biomarkers of inflammation ${ }^{\mathrm{b}}$ in the REGARDS cohort weighted random sample $(n=682)$

\begin{tabular}{|c|c|c|c|c|c|c|c|}
\hline & IL-6 & IL-8 & IL-10 & Fibrinogen & CRP & WBC count & Cys C \\
\hline $\mathrm{OBS}^{\mathrm{c}}$ & -6.4 & -2.9 & 1.9 & -0.2 & -15.3 & 1.1 & -0.9 \\
\hline $95 \% \mathrm{CI}$ & $-11.9,-0.5$ & $-9.4,4.1$ & $-8.1,13.0$ & $-2.7,2.5$ & $-25.6,-3.6$ & $-2.8,5.2$ & $-3.5,1.8$ \\
\hline$p$ value & 0.03 & 0.4 & 0.7 & 0.9 & 0.01 & 0.6 & 0.5 \\
\hline \multicolumn{8}{|l|}{ OBS } \\
\hline Interval $1^{\mathrm{d}}$ & - & - & - & - & - & - & - \\
\hline Interval $2^{\mathrm{d}}$ & 3.7 & 2.0 & 15.3 & 5.7 & -25.1 & -1.3 & -2.3 \\
\hline $95 \% \mathrm{CI}$ & $-18.7,32.3$ & $-22.4,34.1$ & $-23.2,73.0$ & $-4.6,17.1$ & $-55.2,25.2$ & $-14.8,14.5$ & $-12.2,8.6$ \\
\hline Interval $3^{\mathrm{d}}$ & -3.8 & -1.5 & 23.5 & 2.9 & -32.5 & -0.8 & -2.6 \\
\hline $95 \% \mathrm{CI}$ & $-24.4,22.4$ & $-24.9,29.2$ & $-17.4,84.6$ & $-7.0,13.9$ & $-59.5,12.4$ & $-14.4,15.0$ & $-12.4,8.2$ \\
\hline Interval $4^{\mathrm{d}}$ & -7.1 & 1.3 & 17.7 & 3.1 & -39.0 & 3.2 & -3.4 \\
\hline $95 \% \mathrm{CI}$ & $-27.4,18.8$ & $-23.2,33.7$ & $-22.0,77.5$ & $-7.1,14.4$ & $-63.8,2.8$ & $-11.3,20.1$ & $-13.3,7.6$ \\
\hline Interval $5^{\mathrm{d}}$ & -10.1 & -14.0 & 10.5 & 6.7 & -35.7 & -5.7 & -4.8 \\
\hline $95 \% \mathrm{CI}$ & $-33.5,21.6$ & $-38.7,20.5$ & $-33.0,82.3$ & $-5.9,21.0$ & $-65.8,21.1$ & $-21.7,13.7$ & $-16.5,8.4$ \\
\hline$p$ trend & 0.06 & 0.5 & 0.9 & 0.9 & 0.05 & 0.6 & 0.4 \\
\hline
\end{tabular}

OBS, oxidative balance score; IL, interleukin; CRP, C-reactive protein; WBC, white blood cell; Cys C, cystatin C; CI confidence interval. ${ }^{a}$ Adjusted for age, sex, race, region, smoking status, BMI, protein, calories, NSAID, aspirin, and statin use. ${ }^{\mathrm{b}}$ Natural log transformed. ${ }^{\mathrm{c}}$ Estimates represent percent difference per 5 -unit change in OBS. ${ }^{\mathrm{d}}$ Interval 1, OBS 0-5; interval 2, OBS 6-9; interval 3, OBS 10-13; interval 4, OBS 14-17; and interval 5, OBS 18-22. ${ }^{*} p<0.05$ for interval.

than one-fifth (19.7\%) had been diagnosed with diabetes mellitus. Median eGFR was $87 \mathrm{~mL} /$ $\mathrm{min} / 1.73 \mathrm{~m}^{2}$ (interquartile range: $70-99 \mathrm{~mL} / \mathrm{min} / 1.73 \mathrm{~m}^{2}$ ) and median ACR was $7.3 \mathrm{mg} / \mathrm{g}$ (interquartile range: $4.5-14.6 \mathrm{mg} / \mathrm{g}$ ); $22.4 \%$ of the sample had CKD (Table 2).

OBS ranged from 1 to 21 points and the mean score was 11.7 (standard deviation: 3.5). Individuals with higher OBS were generally older (61.3 years in interval 1 vs. 64.0 years in interval $5 ; p=0.02$ ). Current smokers were more likely to be in the lowest interval of OBS (31.8\% in interval 1 vs. $21.9 \%$ in interval $5 ; p<0.001$ ). Those in the lowest OBS interval reported greater energy intakes than those in a higher OBS interval $(2,052 \mathrm{kcal}$ in interval 1 vs. 1,960 kcal in interval 5; $p<0.001$ ) (Table 2).

The Spearman correlation coefficients between OBS and biomarkers of inflammation ranged from $r=-0.09$ to 0.01 , with only CRP $(r=-0.09 ; p=0.02)$ and IL-6 $(r=-0.08 ; p=0.04)$ being significantly correlated with OBS. Among the biomarkers of inflammation, IL- 6 and CRP were strongly correlated with one another $(r=0.50, p<0.001)$, as were fibrinogen and CRP $(r=0.51, p<0.001$ ) (online suppl. Table S1; see www.karger.com/doi/10.1159/000490499 for all online suppl. material).

Unadjusted models of the association between OBS and biomarkers of inflammation were largely null, except for IL-6 and CRP (online suppl. Table S2). In models adjusted for age, sex, race, region, smoking status, BMI, protein intake, energy intake, NSAID, aspirin, and statin use, associations between OBS and IL-6, and OBS and CRP were attenuated but remained significant in the continuous models (Table 3 ). For every 5-unit increase in OBS, the IL-6 concentration was $-6.4 \%$ lower (95\% CI: $-11.9,-0.5 ; p=0.03)$. Comparing the concentration in interval 5 to that in interval 1, the IL-6 concentration was $-10.1 \%$ lower (95\% CI: $-33.5,21.6 ; p$ trend: 0.06). For every 5 -unit increase in OBS, CRP concentration was $-15.3 \%$ lower $(95 \% \mathrm{CI}:-25.6,-3.6$; $p=0.01)$. CRP concentration was $-35.7 \%$ lower $(95 \%$ CI: $-65.8,21.1 ; p$ trend: 0.05 ) when comparing the concentration in interval 5 to that in interval 1. 
Table 4. Adjusted models ${ }^{\mathrm{a}}$ showing the association between OBS and biomarkers of inflammation ${ }^{\mathrm{b}}$, stratified by $\mathrm{CKD}^{\mathrm{c}}$, in the REGARDS cohort weighted random sample $(n=682)$

\begin{tabular}{|c|c|c|c|c|c|c|c|}
\hline & IL-6 & IL-8 & IL-10 & Fibrinogen & CRP & WBC count & Cys C \\
\hline \multicolumn{8}{|l|}{ Interaction } \\
\hline$p$ value $\mathrm{d}^{\mathrm{d}}$ & 0.03 & 0.3 & 0.03 & 0.09 & 0.2 & 0.5 & $<0.001$ \\
\hline \multicolumn{8}{|l|}{$C K D(n=153)$} \\
\hline $\mathrm{OBS}^{\mathrm{e}}$ & -0.1 & 14.7 & -1.3 & 1.7 & -11.4 & 10.7 & 1.7 \\
\hline $95 \%$ CI & $-13.5,15.4$ & $0.3,31.1$ & $-26.7,32.9$ & $-3.7,7.4$ & $-32.4,16.2$ & $-0.6,23.3$ & $-6.6,10.7$ \\
\hline$p$ value & 0.9 & 0.04 & 0.9 & 0.5 & 0.4 & 0.06 & 0.7 \\
\hline \multicolumn{8}{|l|}{ OBS } \\
\hline Interval $1^{\mathrm{f}}$ & - & - & - & - & - & - & - \\
\hline Interval $2^{\mathrm{f}}$ & -7.0 & -2.2 & -32.9 & -13.1 & -35.8 & -7.6 & $-36.1^{*}$ \\
\hline $95 \% \mathrm{CI}$ & $-49.7,71.9$ & $-43.7,69.7$ & $-79.5,120.2$ & $-30.4,8.4$ & $-78.7,93.7$ & $-36.0,33.3$ & $-54.4,-10.4$ \\
\hline Interval $3^{\mathrm{f}}$ & -16.6 & 4.8 & 40.8 & -13.3 & -56.2 & -10.9 & $-37.5^{*}$ \\
\hline $95 \% \mathrm{CI}$ & $-54.1,51.5$ & $-39.3,80.8$ & $-56.5,356.1$ & $-30.3,7.7$ & $-85.3,30.5$ & $-37.5,27.0$ & $-55.3,-12.7$ \\
\hline Interval $4^{\mathrm{f}}$ & -22.3 & 16.9 & -6.8 & -13.6 & -54.6 & 10.8 & $-32.1^{*}$ \\
\hline $95 \% \mathrm{CI}$ & $-57.6,42.3$ & $-32.6,102.9$ & $-71.6,205.7$ & $-30.8,7.7$ & $-84.9,36.9$ & $-23.8,61.2$ & $-51.6,-4.8$ \\
\hline Interval $5^{\mathrm{f}}$ & 31.9 & 59.0 & -16.3 & 2.5 & -35.0 & 4.0 & -26.9 \\
\hline $95 \% \mathrm{CI}$ & $-41.7,198.6$ & $-24.1,233.0$ & $-83.0,311.9$ & $-23.9,38.0$ & $-85.2,185.7$ & $-36.9,71.4$ & $-53.5,15.0$ \\
\hline$p$ for trend & 0.5 & 0.05 & 0.9 & 0.9 & 0.3 & 0.06 & 0.6 \\
\hline \multicolumn{8}{|c|}{ No $C K D(n=529)$} \\
\hline $\mathrm{OBS}^{\mathrm{e}}$ & -10.7 & -3.9 & 7.5 & -1.9 & -19.6 & -0.3 & -1.1 \\
\hline $95 \% \mathrm{CI}$ & $-16.3,-4.7$ & $-10.8,3.6$ & $-3.7,19.9$ & $-4.9,1.0$ & $-30.1,-7.5$ & $-4.3,4.0$ & $-3.1,1.0$ \\
\hline$p$ value & 0.001 & 0.3 & 0.2 & 0.2 & 0.002 & 0.9 & 0.3 \\
\hline \multicolumn{8}{|l|}{ OBS } \\
\hline Interval $1^{\mathrm{f}}$ & - & - & - & - & - & - & - \\
\hline Interval $2^{\mathrm{f}}$ & 4.5 & 0.0 & 36.7 & 6.0 & -37.4 & -9.0 & 6.2 \\
\hline $95 \%$ CI & $-19.3,35.4$ & $-25.8,34.7$ & $-11.5,111.1$ & $-6.1,19.6$ & $-64.2,9.5$ & $-22.2,6.4$ & $-2.3,15.5$ \\
\hline Interval $3^{\mathrm{f}}$ & -4.9 & -0.4 & 48.1 & 0.7 & $-45.0^{*}$ & -8.6 & 4.6 \\
\hline $95 \% \mathrm{CI}$ & $-26.5,23.0$ & $-26.0,34.1$ & $-3.8,128.0$ & $-10.7,13.5$ & $-68.4,-4.1$ & $-21.9,7.0$ & $-3.7,13.6$ \\
\hline Interval $4^{\mathrm{f}}$ & -10.9 & 0.8 & 43.8 & 0.2 & $-50.4^{*}$ & -5.0 & 4.6 \\
\hline $95 \% \mathrm{CI}$ & $-31.4,15.9$ & $-25.6,36.4$ & $-7.4,123.3$ & $-11.4,13.4$ & $-71.9,-12.6$ & $-19.1,11.5$ & $-5.6,11.9$ \\
\hline Interval $5^{\mathrm{f}}$ & -21.0 & -16.8 & 53.5 & 2.6 & $-49.7^{*}$ & -16.2 & 0.9 \\
\hline $95 \%$ CI & $-42.4,8.3$ & $-42.1,19.4$ & $-9.3,159.6$ & $-11.3,18.8$ & $-74.4,-1.3$ & $-31.0,1.7$ & $-8.8,11.5$ \\
\hline$p$ trend & 0.004 & 0.5 & 0.3 & 0.3 & 0.03 & 0.8 & 0.2 \\
\hline
\end{tabular}

OBS, oxidative balance score; IL, interleukin; CRP, C-reactive protein; WBC, white blood cell; Cys C, cystatin C; CKD, chronic kidney disease; CI, confidence interval. ${ }^{a}$ Adjusted for age, sex, race, region, smoking status, BMI, protein, calories, NSAID, aspirin, and statin use. ${ }^{b}$ Natural log-transformed. ${ }^{c}$ CKD was defined as eGFR $<60 \mathrm{~mL} / \mathrm{min} / 1.73 \mathrm{~m}^{2}$ or albuminuria $\geq 30 \mathrm{mg} / \mathrm{g}$. ${ }^{\mathrm{d}} p$ value for interaction based on continuous OBS. ${ }^{\mathrm{e}}$ Estimates represent percent difference per 5-unit change in OBS. ${ }^{\mathrm{f}}$ Interval $1, \mathrm{OBS}$ 0-5; interval 2, OBS 6-9; interval 3, OBS 10-13; interval 4, OBS 14-17; interval 5, OBS 18-22. * $p<0.05$ for interval.

In unadjusted models, there was a difference in the associations of OBS with IL-6, fibrinogen, CRP, and Cys C by CKD status. The unadjusted association of OBS and IL-10 was suggestive of a difference by CKD status, though not statistically significant (online suppl. Table S3).

In adjusted models, interaction by CKD was observed between OBS and IL-6 $(p=0.03)$, OBS and IL-10 ( $p=0.03)$, and OBS and Cys C ( $p<0.001)$ (Table 4$)$. Among those with CKD, the association between a 5 -unit increase in OBS and IL-6 concentration was virtually null $(-0.1 \%$, 95\% CI: $-13.5,15.4 ; p=0.9$ ); IL-6 concentration was $-10.7 \%$ lower $(95 \% \mathrm{CI}:-16.3,-4.7 ; p=$ 0.001 ) for every 5 -unit increase in OBS among those without CKD. These trends persisted across intervals of OBS exposure ( $p$ trend: 0.5 among those with CKD; $p$ trend: 0.004 among those without CKD). 
IL-10 concentration was $-1.3 \%$ lower (95\% CI: $-26.7,32.9 ; p=0.9)$ for every 5 -unit increase in OBS among those with CKD, while the IL-10 concentration was $7.5 \%$ higher $(95 \%$ CI: $-3.7,19.9 ; p=0.2$ ) for every 5 -unit increase in OBS among those without CKD. Similar trends were observed across intervals of OBS exposure ( $p$ trend: 0.9 among those with CKD; $p$ trend: 0.3 among those without CKD).

Among those with CKD, Cys C was 1.7\% higher (95\% CI: -6.6, 10.7; $p=0.7$ ) for every 5 -unit increase in OBS; Cys C concentration was $-1.1 \%$ lower (95\% CI: $-3.1,1.0 ; p=0.3$ ) for every 5 -unit increase in OBS among those without CKD. These trends persisted across intervals of OBS exposure ( $p$ trend: 0.6 among those with CKD; $p$ trend: 0.2 among those without CKD).

While there was no interaction present for the association of OBS with IL-8 by CKD status, examining differences in the associations of OBS and inflammatory markers was an a priori objective. OBS was positively associated with IL-8 among those with CKD; IL-8 was $14.7 \%$ higher (95\% CI: 0.3, 31.1; $p=0.04$ ) for every 5 -unit increase in OBS, and these trends persisted across intervals of OBS exposure ( $p$ trend: 0.05 among those with CKD).

\section{Discussion}

We found that a higher OBS, reflecting an increased antioxidant balance, was associated with lower IL-6 levels and lower CRP levels, but not other studied biomarkers of inflammation. We also examined the associations between OBS and biomarkers of inflammation stratified by CKD (defined as eGFR $<60 \mathrm{~mL} / \mathrm{min} / 1.73 \mathrm{~m}^{2}$ or albuminuria $\geq 30 \mathrm{mg} / \mathrm{g}$ [45]), and found that there is significant interaction by CKD of the association between OBS and IL-6, OBS and IL-10, and OBS and Cys C.

Overall, among those with CKD, there was an inconsistent pattern of associations between OBS and biomarkers of inflammation. The association between OBS and IL- 6 was null among those with CKD, whereas higher OBS was associated with lower IL-6 among those without CKD. Among those without CKD, there was higher IL-10 for higher OBS (unlike other biomarkers of inflammation, higher levels of IL-10 are favorable), compared to those with CKD. Additionally, it was observed that higher OBS was associated with higher Cys C among those with CKD whereas higher OBS was associated with lower Cys $\mathrm{C}$ among those without CKD. Lastly, we found that among those with CKD, OBS was positively associated with IL-8 (a pro-inflammatory cytokine), a finding that was not in the expected direction. This association could potentially be due to changes in diet participants have made to manage their disease. Alternatively, it is possible that some participants with CKD were not prescribed diet changes, particularly in the early stages of CKD, and continued to consume a high fruit and vegetable diet (high in antioxidants). It is conceivable that such a diet in CKD participants might have the opposite of intended effect, due to the presence of nutrients that increase the workload on the kidneys and lead to increased inflammation.

We found that the greater the shift from pro- to antioxidant exposures (low to high OBS), the more favorable the levels of IL- 6 and CRP. As might be expected since IL- 6 induces CRP production [47], IL-6 and CRP were highly correlated and similarly associated with OBS. To our knowledge, this is the first study to examine the association between the balance of proand antioxidants, as measured by OBS, and biomarkers of inflammation among individuals with and without CKD. Previous studies in the REGARDS cohort have found an association between OBS and CRP [4, 20], and this finding is confirmed in this smaller subset of the REGARDS study. A previous study also found an association between OBS and WBC count, although that was not replicated in the present study [20]. While this is not the first study to examine the association between OBS and certain biomarkers of inflammation, it is the first to examine OBS with a wider panel of biomarkers of inflammation and stratified by CKD. 
Additionally, to our knowledge, this study is the first to examine the association between OBS and Cys C. Cys C is a biomarker of inflammation as well as a marker of renal function [48]. As might be anticipated, this study found highly significant interaction by presence or absence of CKD in the association between OBS and Cys C. As with other biomarkers of inflammation, the association between OBS and Cys $\mathrm{C}$ was as predicted among those without CKD (higher OBS associated with more favorable levels of biomarkers of inflammation). The association between OBS and Cys $\mathrm{C}$ was less clear among those with CKD. While Cys $\mathrm{C}$ levels were significantly lower in intervals 2, 3, and 4 compared to interval 1, Cys C levels were almost $2 \%$ higher for every 5 -unit increase in OBS in continuous models.

This study follows up on a previous study in the REGARDS cohort that found that a higher OBS is associated with a lower prevalence of CKD, though the decrease was modest (adjusted odds ratio: 0.93 [95\% CI: 0.86, 0.99]) [19]. While no association was observed between OBS and CKD in adjusted models in this smaller subsample of the REGARDS study, there are a number of reasons for this. The present study uses an exclusively dietary OBS (NSAIDs and aspirin were not included in the OBS, but controlled for in the model instead), uses the residual method of energy adjustment [41] to evaluate OBS components in relation to total energy intake, and simply has a smaller sample size and therefore potentially lacks the power to detect a modest association.

One aim of this study was to determine if the effect of OBS on biomarkers of inflammation differed among those with and without CKD. We found that there was significant interaction by CKD in the IL-6, IL-10, and Cys C models with more pronounced associations observed among those without CKD, and some indication (though nonsignificant) that there was interaction by CKD in the fibrinogen model as well. It is plausible that the association of OBS with biomarkers of inflammation differs by CKD status due to a number of biologic changes that occur during CKD progression [6-13]. While interaction by CKD status was seen for certain biomarkers of inflammation, there was potentially limited power to detect interaction by CKD status for some biomarkers of inflammation, as there were only 153 participants with CKD in our sample. Conversely, it is possible that no interaction was found for certain biomarkers of inflammation because it truly does not exist. Seven different biomarkers of inflammation were analyzed because each represents a particular mechanism and, therefore, we do not necessarily expect all 7 biomarkers to hold the same relationships. Ultimately, further studies are warranted to confirm whether the association of OBS and biomarkers of inflammation differs among those with and without CKD, and research in a cohort of CKD participants is particularly needed to understand the association of OBS with biomarkers of inflammation.

While this is cross-sectional data and the results must be interpreted with caution (e.g., we cannot assess causality), these findings suggest that interventions to improve oxidative balance in the diet might have different effects on inflammation among those with and without CKD. Among those without CKD, increasing antioxidant and decreasing pro-oxidant consumption in the diet could, based on these data, seemingly have the intended effect of lowering inflammation. Alternatively, based on this exploratory analysis, it seems as though the same approach would not be recommended among those with CKD. It should be noted that the CKD population in this study could be highly heterogeneous. Because this is crosssectional data, we do not have information on the severity or duration of CKD, and some individuals may have made changes to their diet because of their CKD that could result in a lower OBS (e.g., eliminating potassium-rich foods would also eliminate some good sources of antioxidants such as oranges and tomatoes). Essentially, in complying with a renal diet that is low in sodium, potassium, and phosphorus, CKD participants may score lower in terms of OBS, though it is difficult to predict how this would impact the association of OBS with biomarkers of inflammation since severity of disease and diet compliance might confound the association. Future research utilizing prospective data is warranted to better understand the association between OBS and biomarkers of inflammation among those with CKD. 
OBS has been validated previously. In the REGARDS cohort, OBS was associated with allcause, cancer, and noncancer morality [49]. OBS has also been validated in a pooled casecontrol study of incident, sporadic colorectal adenoma [18], where it was found that OBS was also associated with circulating biomarkers of oxidative stress (F2-isoprostanes and CRP) [4].

Strengths of this study include the substantial covariate data available and multiple biomarkers of inflammation considered. Additionally, OBS provides a measure that sums the effects of pro- and antioxidant factors in the diet, and this "effect" or "balance" can be used to predict outcomes. Furthermore, by determining a priori the cutoffs for the components of OBS, subjectivity in measurement is reduced [6].

Limitations of this study include the possibility of misclassification due to self-reported data, mainly from the FFQ, which was used to determine the OBS. While dietary questionnaires like the FFQ are subject to recall bias and nutritional intake may be inaccurate, such questionnaires provide acceptable classification of nutrient intake on the population level [50-52]. Given that there were only 153 individuals with CKD, our ability to detect interaction by CKD status may have been limited. Lastly, individuals excluded due to missing data on OBS, CKD, or biomarkers of inflammation were more likely to be black, diabetic, have a lower income, and have a lower level of education, suggesting that selection bias may be a concern.

In conclusion, this study suggests that a higher OBS - consistent with a greater shift from pro- to antioxidant exposures (low to high OBS) - is associated with more favorable levels of biomarkers of inflammation, particularly among those without CKD.

\section{Acknowledgements}

This research project is supported by a cooperative agreement U01 NS041588 from the National Institute of Neurological Disorders and Stroke, National Institutes of Health, Department of Health and Human Services. The content is solely the responsibility of the authors and does not necessarily represent the official views of the National Institute of Neurological Disorders and Stroke or the National Institutes of Health. Representatives of the funding agency have been involved in the review of the manuscript but not directly in the collection, management, analysis or interpretation of the data. The authors thank the other investigators, the staff, and the participants of the REGARDS study for their valuable contributions. A full list of participating REGARDS investigators and institutions can be found at http://www.regardsstudy.org.

\section{Disclosure Statement}

The results presented in this paper have not been published previously, in whole or part, except in abstract format. The authors have no conflict of interest.

\section{Author Contributions}

Study design: D.G.W., O.M.G., M.C., W.M.M., K.J.M., T.J.H., S.E.J. Data analysis: K.J.M., T.J.H., S.E.J. Data interpretation: K.J.M., T.J.H., S.E.J., T.O.I., K.L.C., D.G.W., O.M.G., M.G., M.C. Supervision/mentorship: T.J.H., S.E.J., W.M.M. Each author contributed important intellectual content during manuscript drafting or revision and accepts accountability for the overall work by ensuring that questions pertaining to the accuracy or integrity of any portion of the work are appropriately investigated and resolved. 


\section{References}

1 Sies H: Oxidative stress: oxidants and antioxidants. Exp Physiol 1997;82:291-295.

2 Zhang L, Zalewski A, Liu Y, Mazurek T, Cowan S, Martin JL, Hofmann SM, Vlassara H, Shi Y: Diabetes-induced oxidative stress and low-grade inflammation in porcine coronary arteries. Circulation 2003;108:472-478.

3 Scholz H, Yndestad A, Damås JK, Wæhre T, Tonstad S, Aukrust P, Halvorsen B: 8-isoprostane increases expression of interleukin-8 in human macrophages through activation of mitogen-activated protein kinases. Cardiovasc Res 2003;59:945-954.

4 Kong SYJ, Bostick RM, Flanders WD, McClellan WM, Thyagarajan B, Gross MD, Judd S, Goodman M: Oxidative balance score, colorectal adenoma, and markers of oxidative stress and inflammation. Cancer Epidemiol Biomarkers Prev 2014;23:545-554.

5 Chen X-L, Tummala PE, Olbrych MT, Alexander RW, Medford RM: Angiotensin II induces monocyte chemoattractant protein-1 gene expression in rat vascular smooth muscle cells. Circ Res 1998;83:952-959.

6 Goodman M, Bostick RM, Dash C, Terry P, Flanders WD, Mandel J: A summary measure of pro-and anti-oxidant exposures and risk of incident, sporadic, colorectal adenomas. Cancer Causes Control 2008;19:1051-1064.

7 Cachofeiro V, Goicochea M, De Vinuesa SG, Oubiña P, Lahera V, Luño J: Oxidative stress and inflammation, a link between chronic kidney disease and cardiovascular disease. Kidney Int 2008;74:S4-S9.

8 Tucker PS, Dalbo VJ, Han T, Kingsley MI: Clinical and research markers of oxidative stress in chronic kidney disease. Biomarkers 2013;18:103-115.

9 Reuter S, Gupta SC, Chaturvedi MM, Aggarwal BB: Oxidative stress, inflammation, and cancer: how are they linked? Free Radic Biol Med 2010;49:1603-1616.

10 Oberg BP, McMenamin E, Lucas FL, McMonagle E, Morrow J, Ikizler TA, Himmelfarb J: Increased prevalence of oxidant stress and inflammation in patients with moderate to severe chronic kidney disease. Kidney Int 2004; 65:1009-1016.

11 Aveles PR, Criminácio CR, Gonçalves S, Bignelli AT, Claro LM, Siqueira SS, Nakao LS, Pecoits-Filho R: Association between biomarkers of carbonyl stress with increased systemic inflammatory response in different stages of chronic kidney disease and after renal transplantation. Nephron Clin Pract 2010;116:c294-c299.

12 Stenvinkel P, Holmberg I, Heimbürger O, Diczfalusy U: A study of plasmalogen as an index of oxidative stress in patients with chronic renal failure. Evidence of increased oxidative stress in malnourished patients. Nephrol Dial Transplant 1998;13:2594-2600.

13 Gupta KL, Sahni N: Dietary antioxidents and oxidative stress in predialysis chronic kidney disease patients. J Nephropathol 2012;1:134.

14 Wright ME, Mayne ST, Stolzenberg-Solomon RZ, Li Z, Pietinen P, Taylor PR, Virtamo J, Albanes D: Development of a comprehensive dietary antioxidant index and application to lung cancer risk in a cohort of male smokers. Am J Epidemiol 2004;160:68-76.

15 Van Hoydonck PG, Temme EH, Schouten EG: A dietary oxidative balance score of vitamin C, $\beta$-carotene and iron intakes and mortality risk in male smoking Belgians. J Nutr 2002;132:756-761.

16 Slattery ML, Lundgreen A, Welbourn B, Wolff RK, Corcoran C: Oxidative balance and colon and rectal cancer: interaction of lifestyle factors and genes. Mutat Res 2012;734:30-40.

17 Labadie J, Goodman M, Thyagarajan B, Gross M, Sun Y, Fedirko V, Bostick RM: Associations of oxidative balance-related exposures with incident, sporadic colorectal adenoma according to antioxidant enzyme genotypes. Ann Epidemiol 2013;23:223-226.

18 Dash C, Goodman M, Flanders WD, Mink PJ, McCullough ML, Bostick RM: Using pathway-specific comprehensive exposure scores in epidemiology: application to oxidative balance in a pooled case-control study of incident, sporadic colorectal adenomas. Am J Epidemiol 2013;178:610-624.

19 Ilori TO, Sun Ro Y, Kong SY, Gutierrez OM, Ojo AO, Judd SE, Narayan KV, Goodman M, Plantinga L, McClellan W: Oxidative balance score and chronic kidney disease. Am J Nephrol 2015;42:320-327.

20 Lakkur S, Judd S, Bostick RM, McClellan W, Flanders WD, Stevens VL, Goodman M: Oxidative stress, inflammation, and markers of cardiovascular health. Atherosclerosis 2015;243:38-43.

21 Howard VJ, Cushman M, Pulley L, Gomez CR, Go RC, Prineas RJ, Graham A, Moy CS, Howard G: The reasons for geographic and racial differences in stroke study: objectives and design. Neuroepidemiology 2005;25:135143.

22 Gutiérrez OM, Muntner P, Rizk DV, McClellan WM, Warnock DG, Newby P, Judd SE: Dietary patterns and risk of death and progression to ESRD in individuals with CKD: a cohort study. Am J Kidney Dis 2014;64:204-213.

23 Subar AF, Thompson FE, Kipnis V, Midthune D, Hurwitz P, McNutt S, McIntosh A, Rosenfeld S: Comparative validation of the Block, Willett, and National Cancer Institute food frequency questionnaires: the Eating at America's Table Study. Am J Epidemiol 2001;154:1089-1099.

24 Boucher B, Cotterchio M, Kreiger N, Nadalin V, Block T, Block G: Validity and reliability of the Block98 foodfrequency questionnaire in a sample of Canadian women. Public Health Nutr 2006;9:84-93.

25 Block G, Woods M, Potosky A, Clifford C: Validation of a self-administered diet history questionnaire using multiple diet records. J Clin Epidemiol 1990;43:1327-1335.

26 Caan BJ, Slattery ML, Potter J, Quesenberry CP Jr, Coates AO, Schaffer DM: Comparison of the Block and the Willett self-administered semiquantitative food frequency questionnaires with an interviewer-administered dietary history. Am J Epidemiol 1998;148:1137-1147. 
27 Newby P, Noel SE, Grant R, Judd S, Shikany JM, Ard J: Race and region have independent and synergistic effects on dietary intakes in black and white women. Nutr J 2012;11:25.

28 Cushman M, Judd SE, Howard VJ, Kissela B, Gutiérrez OM, Jenny NS, Ahmed A, Thacker EL, Zakai NA: N-terminal pro-B-type natriuretic peptide and stroke risk: the reason for geographic and racial differences in stroke cohort. Stroke 2014;45:1646-1650.

29 Alexander KS, Zakai NA, Gillett S, McClure LA, Wadley V, Unverzagt F, Cushman M: ABO blood type, factor VIII, and incident cognitive impairment in the REGARDS cohort. Neurology 2014;83:1271-1276.

30 Goodman M, Bostick RM, Dash C, Flanders WD, Mandel JS: Hypothesis: oxidative stress score as a combined measure of pro-oxidant and antioxidant exposures. Ann Epidemiol 2007;17:394-399.

31 Stengel B, Couchoud C, Cénée S, Hémon D: Age, blood pressure and smoking effects on chronic renal failure in primary glomerular nephropathies. Kidney Int 2000;57:2519-2526.

32 Shankar A, Klein R, Klein BE: The association among smoking, heavy drinking, and chronic kidney disease. Am J Epidemiol 2006;164:263-271.

33 Orth SR, Stöckmann A, Conradt C, Ritz E, Ferro M, Kreusser W, Piccoli G, Rambausek M, Roccatello D, Schäfer K: Smoking as a risk factor for end-stage renal failure in men with primary renal disease. Kidney Int 1998;54: 926-931.

34 Lash JP, Go AS, Appel LJ, He J, Ojo A, Rahman M, Townsend RR, Xie D, Cifelli D, Cohan J: Chronic Renal Insufficiency Cohort (CRIC) Study: baseline characteristics and associations with kidney function. Clin J Am Soc Nephrol 2009;4:1302-1311.

35 Kronborg J, Solbu M, Njølstad I, Toft I, Eriksen BO, Jenssen T: Predictors of change in estimated GFR: a population-based 7-year follow-up from the Tromsø study. Nephrol Dial Transplant 2008;23:2818-2826.

36 Hallan SI, Orth SR: Smoking is a risk factor in the progression to kidney failure. Kidney Int 2011;80:516-523.

37 National Kidney Foundation: Kidney disease outcomes quality initiative (K/DOQI). https://www.kidney.org/ professionals/guidelines.

38 Goroll AH, Mulley AG: Primary Care Medicine: Office Evaluation and Management of the Adult Patient. Philadelphia, Lippincott Williams and Wilkins, 2012.

39 Fiebach NH, Barker LR, Burton JR, Zieve PD: Principles of Ambulatory Medicine. Philadelphia, Lippincott Williams and Wilkins, 2007.

40 Fauci AS: Harrison's Principles of Internal Medicine. New York, McGraw-Hill, Medical Publishing Division, 2008.

41 Willett W, Stampfer MJ: Total energy intake: implications for epidemiologic analyses. Am J Epidemiol 1986; 124:17-27.

42 Gillett SR, Boyle RH, Zakai NA, McClure LA, Jenny NS, Cushman M: Validating laboratory results in a national observational cohort study without field centers: the Reasons for Geographic and Racial Differences in Stroke cohort. Clin Biochem 2014;47:243-246.

43 Cushman M, McClure LA, Howard VJ, Jenny NS, Lakoski SG, Howard G: Implications of increased C-reactive protein for cardiovascular risk stratification in black and white men and women in the US. Clin Chem 2009; 55:1627-1636.

44 Zakai NA, McClure LA, Prineas R, Howard G, McClellan W, Holmes CE, Newsome BB, Warnock DG, Audhya P, Cushman M: Correlates of anemia in American blacks and whites: the REGARDS Renal Ancillary Study. Am J Epidemiol 2008;169:355-364.

45 Kidney Disease Improving Global Outcomes (KDIGO): KDIGO clinical practice guideline for the evaluation and management of chronic kidney disease. Kidney Int Suppl 2013;3:1-150.

46 Levey AS, Stevens LA, Schmid CH, Zhang YL, Castro AF, Feldman HI, Kusek JW, Eggers P, Van Lente F, Greene T: A new equation to estimate glomerular filtration rate. Ann Intern Med 2009;150:604-612.

47 Pepys MB, Hirschfield GM: C-reactive protein: a critical update. J Clin Invest 2003;111:1805-1812.

48 Peralta CA, Shlipak MG, Judd S, Cushman M, McClellan W, Zakai NA, Safford MM, Zhang X, Muntner P, Warnock D: Detection of chronic kidney disease with creatinine, cystatin $C$, and urine albumin-to-creatinine ratio and association with progression to end-stage renal disease and mortality. JAMA 2011;305:1545-1552.

49 Kong SY, Goodman M, Judd S, Bostick RM, Flanders WD, McClellan W: Oxidative balance score as predictor of all-cause, cancer, and noncancer mortality in a biracial US cohort. Ann Epidemiol 2015;25:256-262.e251.

50 Toft U, Kristoffersen L, Ladelund S, Bysted A, Jakobsen J, Lau C, Jørgensen T, Borch-Johnsen K, Ovesen L: Relative validity of a food frequency questionnaire used in the Inter99 study. Eur J Clin Nutr 2008;62:1038.

51 Judd SE, Aaron KJ, Letter AJ, Muntner P, Jenny NS, Campbell RC, Kabagambe EK, Levitan EB, Levine DA, Shikany JM: High sodium: potassium intake ratio increases the risk for all-cause mortality: the REasons for Geographic And Racial Differences in Stroke (REGARDS) study. J Nutr Sci 2013;2:e13.

52 Dyer A, Elliott P, Chee D, Stamler J: Urinary biochemical markers of dietary intake in the INTERSALT study. Am J Clin Nutr 1997;65:1246S-1253S. 